BRIEF REPORT

\title{
Electromagnetic Interference From Electronic Devices Used in the Management of Type 1 Diabetes Can Impair the Performance of an Avalanche Transceiver in Search Mode
}

\author{
Steven C.M. Miller, BSc, MBChB, PhD, FRCP, FRACP \\ From the Departments of Endocrinology, Diabetes, and Internal Medicine, North Shore Hospital, Auckland, New Zealand.
}

\begin{abstract}
Objective.-Portable electronic devices play an important role in the management of type 1 diabetes mellitus. Electromagnetic interference from electronic devices has been shown to impair the function of an avalanche transceiver in search mode (but not in transmitting mode). This study investigates the influence of electromagnetic interference from diabetes devices on a searching avalanche beacon.

Methods.-The greatest distance at which an avalanche transceiver (in search mode) could accurately indicate the location of a transmitting transceiver was assessed when portable electronic devices (including an insulin pump and commonly used real-time continuous subcutaneous glucose monitoring system [rtCGMS]) were held in close proximity to each transceiver.

Results.-The searching transceiver could accurately locate a transmitted signal at a distance of $30 \mathrm{~m}$ when used alone. This distance was unchanged by the Dexcom G4 rtCGMS, but was reduced to $10 \mathrm{~m}$ when the Medtronic Guardian rtCGMS was held close (within $30 \mathrm{~cm}$ ) to the receiving beacon. Interference from the Animas Vibe insulin pump reduced this distance to $5 \mathrm{~m}$, impairing the searching transceiver in a manner identical to the effect of a cell phone.

Conclusions.-Electromagnetic interference produced by some diabetes devices when held within 30 $\mathrm{cm}$ of a searching avalanche transceiver can impair the ability to locate a signal. Such interference could significantly compromise the outcome of a companion rescue scenario. Further investigation using other pumps and rtCGMS devices is required to evaluate all available diabetes electronics. Meantime, all electronic diabetes devices including rtCGMS and insulin pumps should not be used within $30 \mathrm{~cm}$ of an avalanche transceiver.
\end{abstract}

Key words: type 1 diabetes mellitus, T1DM, avalanche transceiver, avalanche beacon, electromagnetic interference

\section{Introduction}

The avalanche transceiver (or beacon) is considered to be an essential component of backcountry safety equipment and should be carried by all persons who venture into avalanche-prone terrain. Although there is evidence to show that use of an avalanche transceiver can reduce duration of burial and improve mortality among avalanche victims, ${ }^{1}$ observational studies have demonstrated variable adherence to avalanche transceiver use among backcountry recreationalists. In both the United States

Corresponding author: Steven C. M. Miller, PhD, North Shore Hospital, Shakespeare Road, Takapuna, Auckland 0629, New Zealand (e-mail: steven@endocrinology.co.nz). and Europe, avalanche transceiver use has been reported by $11 \%$ to $98 \%$ of persons upon entry into the backcountry. ${ }^{2,3}$ Those participating in backcountry skiing $(89 \%$ to $98 \%)$ are significantly more likely to carry a transceiver than backcountry snowboarders (90\%), snowmobilers $(57 \%)$, and those using snowshoes $(11 \%$ to $25 \%$ ). Of considerable concern are persons who access the backcountry from within a resort area, with only $9 \%$ to $36 \%$ avalanche beacon use reported at point of entry into avalanche-prone terrain.

The influence of avalanche transceivers on life-saving medical electronic devices has been investigated previously. Although interference has been observed during telemetric communication with permanent cardiac pacemakers or implantable cardiac defibrillators, the function 
of the pacemaker or implantable cardiac defibrillator itself is unaffected by the use of an avalanche transceiver when both devices are in close proximity. ${ }^{4}$ Conversely, avalanche transceivers have themselves been shown to be susceptible to electromagnetic interference from commonly used personal electronic equipment such as global positioning satellite (GPS) locators, cell phones, MP3 players, and two-way radios when these devices are held adjacent to a transceiver. ${ }^{5}$ The signal transmitted by a transceiver is not affected; however, the ability of an avalanche transceiver in search mode to accurately determine the proximity and direction of a transmitting transceiver can be significantly impaired. These observations have led to the recommendation that all portable electronic devices be kept at least $30 \mathrm{~cm}$ away from an avalanche transceiver while in search mode so that a companion rescue scenario is not compromised.

Portable electronic devices play an important role in the management of type 1 diabetes mellitus. ${ }^{6}$ A real-time continuous subcutaneous glucose monitoring system (rtCGMS) gives the user a continuous estimation of interstitial fluid glucose concentration (which approximates that of blood glucose). A thin wire sensor inserted into the subcutaneous interstitial space (usually in the abdomen) is attached to a small transmitter and worn for as long as 2 weeks at a time. Every 5 minutes or so, the interstitial fluid glucose concentration is determined and transmitted wirelessly to a handheld receiver that informs the user of the current reading. An insulin pump constantly delivers rapid-acting analogue insulin at a variable rate as determined by the user. Often, an rtCGMS and insulin pump are used together, with some devices being able to communicate with each other. Together, these devices allow the experienced operator to maintain near-normal glycemic control (and are likely to become more prevalent with time ${ }^{7}$ ).

With appropriate preparation, a diagnosis of type 1 diabetes should not be seen as an impediment to participation in backcountry activities. Accordingly, some persons with type 1 diabetes who use an insulin pump and rtCGMS will also use avalanche transceivers while in the backcountry, in line with expert recommendation. The influence of electromagnetic interference from an insulin pump and rtCGMS on the performance of avalanche transceivers is unknown, and was assessed.

\section{Methods}

In an open field (away from buildings and overhead or underground structures), the performance of two widely available and commonly used triple antenna digital avalanche transceivers-the Mammut Pulse Barryvox (Mammut Sports Group, Switzerland), and the Arva 3
Axes (Arva Nic-Impex, Annecy, France)-was investigated. The ability of each transceiver in search mode to determine the proximity and direction to the other (in transmit mode) was examined at set distances $(45 \mathrm{~m}, 30$ $\mathrm{m}, 15 \mathrm{~m}, 10 \mathrm{~m}, 5 \mathrm{~m}$, and $1 \mathrm{~m})$. The most distant point at which the receiving transceiver was able to accurately determine both the distance from and direction to the transmitting (source) beacon was recorded (effective search range). Both transceivers were investigated in digital mode.

In turn, portable electronic devices-Baofeng UV-5R two-way radio (Baofeng, Quanzhou, China), iPhone 4 and iPhone 5 (Apple, Cupertino, CA, USA), Dexcom G4 rtCGMS (Dexcom, San Diego, CA, USA), Medtronic Guardian rtCGMS (Medtronic, Minneapolis, MN, USA), and Animas Vibe insulin pump (Animas, West Chester, PA, USA) - were then held underneath either the transmitting or receiving beacon, and the recordings were repeated to assess the influence of the electronic device on transceiver function. All devices (including the avalanche beacons) had either fresh batteries or a more than $95 \%$ charge.

\section{Results}

The greatest distance at which the searching transceiver could accurately determine the direction to and distance from the transmitting transceiver was $30 \mathrm{~m}$. Identical results were obtained when either avalanche transceiver was used in the transmitting or searching position. There was no interference observed when any electronic device was in close proximity to the transmitting transceiver or more than $30 \mathrm{~cm}$ away from the searching transceiver. Avalanche transceivers did not influence the function of the diabetes devices (data not shown).

When the electronic devices were held within $30 \mathrm{~cm}$ of the searching transceiver, the distance at which the searching transceiver could accurately determine the direction to and distance from the transmitting transceiver was reduced (Figure). The magnitude of effect was different depending on which device was used. The devices that impaired beacon function most were the iPhone 4, the iPhone 5, and the Animas Vibe pump. These all reduced the transceiver's effective search range to just $5 \mathrm{~m}$. The Dexcom G4 rtCGMS did not produce any apparent interference.

\section{Discussion}

These data confirm that electronic diabetes devices can interfere with avalanche transceiver function. The cause of this effect is presumed to be electromagnetic interference. The resulting reduction of the transceiver's search range from $30 \mathrm{~m}$ to $5 \mathrm{~m}$ is significant, and has potential to delay recovery of a buried avalanche victim (and thus 


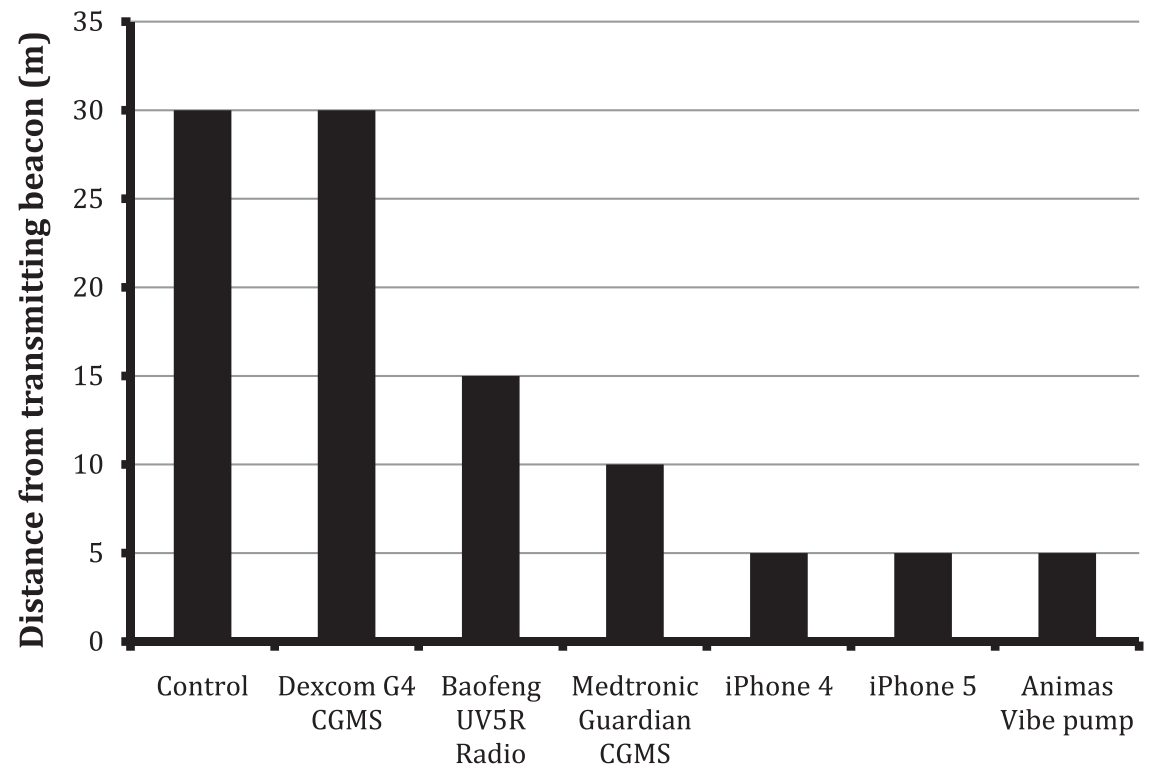

Figure. Avalanche transceiver performance when in close proximity to personal electronic devices. The vertical axis indicates the distance (in meters) from the transmitting transceiver when the device indicated on the horizontal axis is held underneath the receiving transceiver in search mode. "Control" indicates results obtained with no interfering personal electronic device. Results were the same regardless of which avalanche transceiver was transmitting or searching, and no effect was seen when the device was held more than $30 \mathrm{~cm}$ from the receiving transceiver. Data reflect the mean of 3 identical experiments. CGMS, continuous glucose monitoring system.

reduce chances of the victim's survival). Therefore, this important information should be shared with persons (and their advising physicians) with type 1 diabetes who also use an avalanche transceiver.

In these experiments, the Animas Vibe insulin pump produced the most interference. That may be due to the presence of moving parts (electric pump motor). The Dexcom G4 rtCGMS did not produce any electromagnetic interference, whereas the other rtCGMS examined (Medtronic Guardian) caused an intermediate level of interference. It is, therefore, not safe to assume that all devices of similar function will result in identical interference effects. A more comprehensive analysis of all available continuous glucose monitoring systems and insulin pumps (with additional avalanche transceivers) would be required to fully appreciate the extent of this effect.

The magnitude of effect was similar to that observed (and previously reported) with other commonly used electronics (such as cell phones, MP3 players, and so forth). Diabetes electronic devices per se, therefore, do not lead to additional risk compared with other portable electronics when they are used alongside avalanche transceivers alone. This study did not examine the combined effect of multiple devices simultaneously in close proximity to an avalanche beacon. As a person with type 1 diabetes may carry several devices (eg, insulin pump and continuous glucose monitoring system in addition to cell phone, radio, GPS, MP3 player, and so forth), the potential effects of electromagnetic interference on an avalanche transceiver may be additive. This research question warrants further investigation.

No interference was observed when the electronic diabetes device was held more than $30 \mathrm{~cm}$ from the receiving beacon, which is in line with current recommendations for other personal electronic devices (15 to $50 \mathrm{~cm})$. This study would, therefore, recommend that persons with type 1 diabetes be advised to keep their rtCGMS or insulin pump $30 \mathrm{~cm}$ or more from an avalanche transceiver while in avalanche-prone terrain in the backcountry.

\section{Acknowledgments}

I would like to acknowledge New Zealand Medical and Scientific, Auckland, New Zealand, who provided the Dexcom G4 rtCGMS and Animas Vibe pump for use in this study.

\section{References}

1. Hohlrieder M, Mair P, Wuertl W, Brugger H. The impact of avalanche transceivers on mortality from avalanche accidents. High Alt Med Biol. 2005;6:72-77.

2. Silverton NA, McIntosh SE, Kim HS. Avalanche safety practices in Utah. Wilderness Environ Med. 2007;18:264-270.

3. Procter E, Strapazzon G, Dal Cappello T, Castlunger L, Staffler HP, Brugger H. Adherence of backcountry winter 
recreationists to avalanche prevention and safety practices in northern Italy. Scand J Med Sci Sports. 2013;24:823-829.

4. Dorenkamp M, Blaschke F, Voigt K, Fleck E, Goetze S, Roser M. Electromagnetic interference of avalanche transceivers with cardiac pacemakers and implantable cardioverter defibrillators. Pacing Clin Electrophysiol. 2013;36: 931-938.

5. Barkhausen J. The effect of external interference on avalanche transceiver functionality. Proceedings of the
2012 International Snow Science Workshop; 2012 Sept 16-21; Anchorage, AK.

6. Siegmund T, Kolassa R, Thomas A. Clinical update on insulin pump therapy in combination with continuous glucose monitoring. Minerva Endocrinol. 2013;38:133143.

7. Lodwig V, Kulzer B, Schnell O, Heinemann L. What are the next steps in continuous glucose monitoring? J Diabetes Sci Technol. 2014;8:397-402. 